J. Bio-Sci. 29(1): 153-161, 2021 (June)

ISSN 1023-8654

http://www.banglajol.info/index.php/JBS/index

DOI: https://doi.org/10.3329/jbs.v29i0.54831

\title{
THE UNDERSTANDING OF ENIGMAS RELATED TO CANCER: CANCER ORIGIN AND PROGRESSION- A REVIEW
}

\author{
MT Hasan* \\ Biotechnology and Genetic Engineering Discipline, Khulna University, Bangladesh
}

\begin{abstract}
Human body is an excellent example of self-synchronized system. This coordination is existing between organs, cells, subcellular organels even in molecules by which these are formed and maintaining a subtle pattern of morphogenesis. Although having same responsible genes for metamorphosis of different organisms, the expression pattern of those genes varies in every species. Frequent repetition of a pathway energizes the pathway to be established itself as an ordinary and stable event in that developmental procedure. Cancer can be called a disease of geometry which is also metastasize in human body maintain a developmental pathway. The paradoxes in cancer cannot be explained with the conventional mechanistic approaches; therefore, it requires a holistic approach (Morphogenetic Field) for better understanding. Field effects of various epigenetic factors such as heavy metals and radiations affect the normal developmental pathway of organogenesis and energize the progression of cancer. Quantum field along with gravitational field also interfere with the self-organizing process of a biosystem and have influence on the mutation of different protooncogenes. Understanding of this field concept is very promising for achieving advancement in the various emerging fields of biology like optogenetics, bioinformatics, computational biology and cybernetics etc. In true sense, the morphogenetic field concept offers an exciting new canvas like checkpoint therapy in cancer prognosis.
\end{abstract}

Key words: Developmental Pathway, Epigenetic field, Metamorphosis, Morphogenetic field, Quantum field

\section{Introduction}

Various types of views tried to encrypt the enigma of the development of cancer in human body but it still hiding its spell and holding the same position over many years after year in the history of human civilization, in spite of having an advanced medical knowledge. We may describe life as an information processor that has the ability to self-organize, driven by the action of consciousness and obviously including the surrounding environment to form the totality of reality. On accord to the theory of clonal selection, abnormal cancer cells survive and proliferate by being selected in the evolutionary pathway through environmental influence, inheritance or spontaneous error (Breivik 2005). To understand the nature of cancer a paradigm shift towards a stochastic realization of biology is required. Every disease can be differentiated from other one is because of their own developing pattern. Cancer also has its distinctive developing patterns and this is why we need to enrich our knowledge by understanding the metamorphosis of cancer by studying its developmental pattern. So in regard to cancer which is now treating as an abnormal organ (Reya et al. 2001) we might

*Author for correspondence: tasnimul.hasan1993@gmail.com 
consider it as unprecedented and abnormal whole in the complex hierarchy which work in the biological organization and able to explain cancer with a holistic paradigm viewpoint.

\section{Morphogenetic field concept}

Morphic resonance presents a revolutionary information field understanding of the nature and evolution of life, acquaintance with it is an essential part of new paradigm scientific literacy (Ervin Laszlo 1996). In spite of having the same genes in their metamorphosis it is their form which defining them as different individuals (Sheldrake 2005). It is the morphogentic fields (it is a group of cells able to respond to discrete, localized biochemical signals leading to the development of specific morphological structures), the organizing fields of molecules, crystals, cells, tissues, indeed all biological systems (Sheldrake 2005). Thus, beyond all question of quantity there lie questions of pattern, which are essential for the understanding of nature (Whitehead 1934). The active process of maintaining a complex morphology may likewise be relevant to suppression of cancerous disorganization in favor of cellular activities that aligned within the morphological needs of the host organism (Levin 2012).

\section{Sub-fields of morphogenetic field}

Morphogenetic field considered as similar to the known fields of physics in that they are capable of ordering physical changes, even though they themselves cannot observe directly. Gravitational field, Electric field, Magnetic field, Epigenetic field, Quantum field and Energetic field etc along with many unknown fields those are involved in the spatial structures are invisible, intangible, inaudible, tasteless and odorless they are only detectable only through their respective field effects (Sheldrake 2005). These above mentioned fields comprising the morphogenetic field with the accumulative effects of each field and emerging a hierarchial organization. Fig. 1 showing the various sub fields of morphogenetic field, which is responsible for complex hierarchial organization of organisms

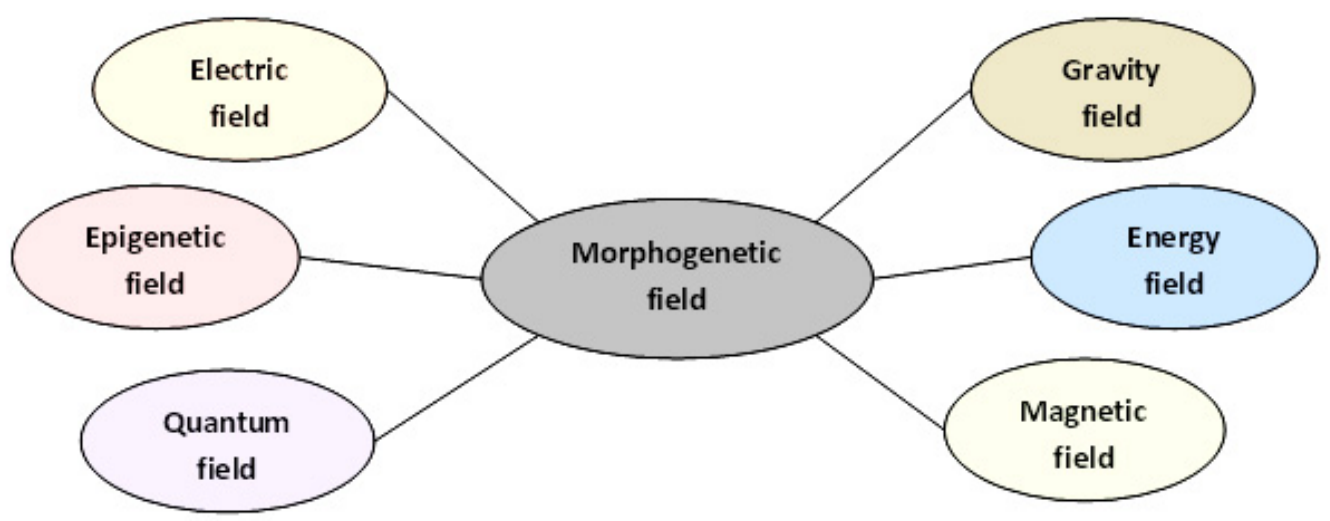

Fig. 1: Schematic representation of the sub-fields of morphogenetic field

\section{Morphic unit}

The functional unit of morphogenetic field is 'morphogenetic germ' or 'holon' (Sheldrake 2005). It has been described the complexity of life by using the term 'holon' that was first introduced by Arthur Koestler (1967), 
in his book 'The Ghost in the Machine'. It can be explained with an example; we may consider individual cells as autopoietic holon comprising the organs which are again act as holon for creating the organismic holon. This is the holarcy of holon and which is the basis of life (Sahtouris 1999) and with a certain level of hierarchy these creating the individual who is capable of thinking, perceiving and making decisions.

\section{The information carried by the morphogenetic field}

\section{Subtle pre-patterns}

A recent paper characterized in real time that the bioelectric properties of a highly dynamic morphogenetic event e. g. the formation of face in Xenopus laevis embryos by using voltage-reporter dyes and time-lapse microscopy a movie was made of the many dynamic changes occurring at this time in the distribution of cells with distinct transmembrane potentials which revealing a rich regionalization of voltage gradient that demarcates the interior of the neural tube, the future mouth and thin bilateral crescents on the edge of the face (Vandenberg et al. 2011, Levin 2012). This quantitative spatio-temporal profiling of native physiology along with detailed characterization of anatomical and molecular-genetic perturbation of the boundaries of the hyperpolarization domains, is a superb example of physiology serving as a subtle pre-pattern for regions of gene expression, much as transcriptional states act as pre-patterns for subsequent anatomy (Levin 2012).

\section{Positional information}

Positional information is the information required to integrate cell activity into a system (Furusawa and Kaneko 2003) which enables cells and tissues to discern their location relative to each other within a complex 3-dimensional structure. The dynamic nature of the storage positional information in morphogenetic fields offers a major area of future investigation (Levin 2012).

\section{Information about modification beyond transcriptional network}

The future behavior of cells crucially depends on the geometric shape of its substrate (Huang and Ingber 2000); this geometric shape is an ideal signal, which cannot describe by genetic, and proteomic profiling. Moreover the physical properties can serve same functions include mechanical properties of tissues (Beloussov et al. 2000, Discher et al. 2005), ultra-weak photon emission (Beloussov 2001, Popp 2003), and bioelectric gradients (Levin 2007). Therefore, the modification of the gene expression at transcriptional level, actually indicate the epigenetic influence over the gene expression.

\section{Positional information of cancer cells, interpreting the effect of morphogenetic field}

A case study is the transplantation of rat testis to the spleen induces formation of interstitial cell tumors (Biskind 1945), and also normal rat ovary tissue put into normal rat spleen results a malignant neoplasm (Biskind 1944), implantation of mouse embryos into adults causes teratocarcinomas (Stevens 1970) and these because of an interference of the host and implanted morphogenetic field structure. These cases are proving that disruption of normal topographical tissue relationships tend to induce cancer (Liven 2012).

\section{Tumors appear to be normal by its developmental patterning}

Tumor cells integrated into wild type embryonic hosts that integrated as normal tissue (Astigiano et al. 2005). Human metastatic melanoma cells injected into zebrafish embryos acquire a non-neoplastic phenotype, but form tumor when injected into zebrafish after organogenesis (Haldi et al. 2006). These data are consistent with the morphogenetic field concept because they indicate the power of activity patterning cues to normalize cancer cues (Liven 2012). 


\section{Normalization of cancer by regeneration}

Highly-regenerative organisms are resistance to tumors (Brockes 1998) and this inverse relationship between regeneration and cancer susceptibility (Breedis 1952, Prehn 1997) is more compatible with the importance of morphogenetic field guidance than a focus on cancer risk associated with the presence of highly-active, undifferentiated cells (Levin 2012). Amphibian limb regeneration can normalize tumors (Needham 1936). Thus the normalization of tumors by active remodeling represents one of the most profound and exciting areas for future work in understanding morphogenetic fields and their interpretation by growing tissue (Levin 2012).

\section{Role of chronic psychological stress in the progression of cancer from morphogenetic aspect}

There is a biomolecular basis for the role of chronic psychological stress in development of cancer (Sobhani et al. 2012). Chronic psychological stress called carcinogen and can induce cancer through quantum fluctuation (Mamun 2014). Psychological stress increases the glucocorticoid level through the Hypothalamic Pituitary Adrenocortical (HPA). When any external events evaluated as threatening or stressful, this activate cortical and limbic structures of the central nervous system and ultimately activate the HPA axis.

\section{Mechanism of glucocorticoid action on cytokine suppression}

An increased level of glucocorticoid under chronic stress may exert effect on immune response genes at transcriptional level resulting low production of cytokines. GCs exert their effects through induction of specific GC-regulated genes, GILZ (GC-induced leucine zipper) and GITR (GC-induced TNFR family-related). GILZ is expressed constitutively in normal cells and up regulated by GCs. GILZ supposedly mediated a number of GC effects, including modulation of activation-induced cell death and antagonism of transcription factors. GILZ showed to act by inhibiting NF-KB translocation and hence it is binding to the IKB DNA site through a protein, thereby inhibiting the translocation of AP-1 and subsequently its DNA binding. Thus, GC triggering no transcription of cytokine genes enabling the tumor cells to survive.

\section{Suppression of Nk cell activity through glucocorticoid in aspect of morphogenetic field by epigenetic modification}

Psychological stress reduces the activity of natural killer cell and dysregulates cytokine balance is now well established which is mediated by stress induced release of glucocorticoids. Epigenetic modification, induced by GCs, appears to be one mechanism by which NK cell function is dysregulated. GCs have shown to upregulate the histone acetyltransferase activity and down-regulate the histone deacetylase activity. NK cells express a variety of molecules including IFN, TNF-a, IL-6, perforin, granzyme B, lymphocyte adhesion molecules (e.g. LFA-1), NK cell activating receptor molecules (e.g. NKP-30), etc. During chronic stress GCs have shown to down-regulate the expression of all of these effectors and thereby stimulating cancer progression.

\section{Mechanism of chronic stress induced glucocorticoid mediating suppression of P53}

P53 is a transcription factor that regulates many genes including p p21, Bax, Mdm2, GADD45, etc, which play effective role against cancer. The tumor suppressor $p^{53}$ frequently inactivated in human cancer most commonly by mutation, but also by other mechanisms including over expression of inhibitory proteins and sequestration in the cytoplasm. GC activated GR can bind with $\mathrm{p}^{53}$ in the cytoplasm and thus inhibition of $\mathrm{p}^{53}$ to translocate into nucleus occurs through sequestration in the cytoplasm. In breast and other cancer, GC induces the expression of genes such as Bcl-2, Bak, SGK-1 and MKP-1 frequently associated with protection against cell apoptosis. Thus, GC preventing the $p^{53}$ mediated apoptosis. Therefore, GC reduces the $p^{53}$ mediated activities, which in turn facilitate the cancer progression. 


\section{Role of quantum field in cancer from morphogenetic viewpoint}

According to the Schrodinger (1944), "The mutations are actually due to quantum jumps in the gene molecule and the 'quantum jump' which means the transition from one relatively stable molecular configuration to another". The idea of directed or adaptive mutation suggests that, consciousness may play a direct role in evolution (Goswami and Todd 1997). Then it might be apparent to say the inverse quantum zeno effect is analogous to the canalized pathway of change (Waddington 1957) which was proposed for the extension of unitary field concept (morphogenetic field) in order to cover the temporal aspect of development (Sheldrake 1981). The wave functions collapse and genetic material became fixing in one configuration only when consciousness sees a meaningful pattern emerge that merit phenotypical expression and genetic information alone cannot predict the outcomes (Goswami and Todd 1997). Quantum gravitational effects may induce stochastic fluctuations in the structure of space-time (Aloiso et al. 2004). This is the reason behind increasing instability of genome and proteome in cancer and accounts strongly for drug resistance.

\section{Heavy metals induced cancer in the aspect of morphogenetic field}

The carcinogenicity of heavy metals reflecting an epigenetic mechanism such as chromatin condensation and subsequent DNA hypermethylation induced gene silencing of tumor suppressor genes (Shamy et al. 2013). Moreover, heavy metals can induce the synthesis of miR-222, which is capable of turn off the expression of tumor suppressor genes like p-27, p-57 and PTEN, and as these genes play important role in cell cycle, silencing of these genes lead to rapid proliferation of cancer cells (Zhou et al. 2013). Again, the generation of reactive oxygen species by the heavy metals leading to oxidative damage of cellular metabolic enzymes, lipids, carbohydrates, repair mechanisms and other regulatory proteins that result in an alteration of gene expression (Yan et al. 2003, Kampa and Castanas 2008).

\section{Molecular mechanism of radiation induced cancer in aspect of morphogenetic field}

One of the sub-fields of morphogenetic field is electromagnetic field e.g. raido-frequency modulated electromagnetic fields may induce cancer such as brain cancer by disturbing in the function of blood brain barrier (Joenvaara et al. 2002). They gave a hypiothetical model to explain the mechanism of radiation induced brain cancer. This model states that brain tissue- nerve cells and endothelial cells lining capillary blood vessels absorb radiations. With an unidentified mechanism, it activates cellular stress kinase either directly or indirectly. This activation of stress response is caused by either activation of p-38 MAPK cascade alone or also some other kinases located upstream/ downstream of p-38 MAPK which leads to phosphorylation of hsp-27. The phosphorylated hsp-27 stabilizes endothelial cell stress fibers and in conjunction with estrogen, alters the generation/ secretion of bEGF. This causes the increase of blood-brain barrier permeability. In reaction the endothelial cells shape change and formation of gaps between cells; increased pinpocytosis through endothelial monolayer; obstruction of capillary blood flow by bleb formation on the lumen surface of endothelial cells- this event could increase blood pressure locally and force to large molecules to pass through endothelial cells. The phosphorylated hsp-27 forms a complex apoptosome, which prevents activation of procaspase- 9 and subsequently prevents the activation of procaspase- 3 and inhibit caspase-3 dependent apoptosis pathway (Jeonvaara et al. 2002).

\section{Proposed pathway of origin and progression of cancer from morphogenetic viewpoint}

Fig. 2 is a hypothetical pathway of origin and progression of caner where the subtle pattern of morphogenesis influenced by various environmental hazardous elements likes different kinds of radiation, heavy metals, psychological stress etc. These factors with field effects are affecting the inheritance pattern of an organism and creating disturbances in the normal canalized pathway of metamorphosis of living organisms. 


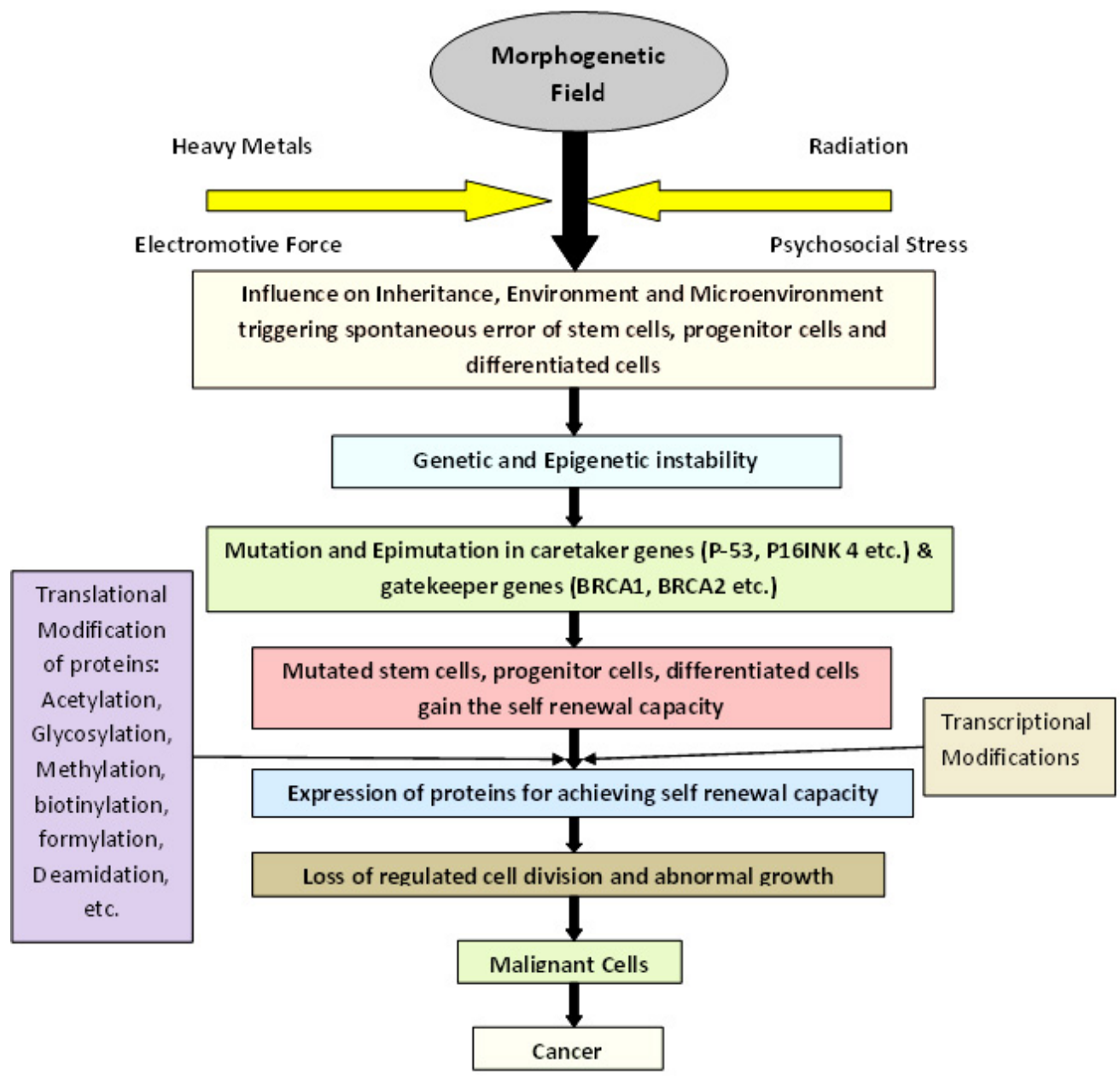

Fig. 2: Proposed pathway of cancer progression from morphogenetic viewpoint

Hence, for the changes in the inheritance pattern, environment and microenvironment of stem cells, progenitor cells and differentiated cells of living organisms spontaneous error occurs which resulting into genetic and epigenetic instability. Consequently, mutations and epi-mutations take place in caretaker genes and gatekeeper genes. The mutated stem cells, progenitor cells and differentiated cells next achieve the selfrenewal capacity and express proteins for energizing the self-renewal pathway and inhibiting the pathway of programmed cell death of those regarding cells. The self-renewal property of a cancer cell adorned with very spooky mechanisms like modifications of the expressed proteins by various types of translational modifications. All of these events help a cancer cell to loss the power of control over cell division and contact inhibition. Thus, normal cells are converting into malignant cells with the power of abnormal growth. 


\section{Discussion}

Cancer is a disease of geometry, caused by an escape from the control of the morphogenetic field. The field plays a crucial role in the operation of life and it could be an account for a holistic explanation of cancer. The formation of tumor initiated when the cells stop obeying the normal patterning cures of the body. The tumor cells gradually appear to be normal in the metamorphosis pathway or normalization of cancer cells inside our body emphasizes the necessity of understanding the concept about canalized pathway studied in morphogenetic field. So the morphogenetic field model proposed can answer the basic flaws in biology but it must acknowledged that it is a very tiny step towards the vast arena of cancer mystery along with the gorgeous and enormous morphogenetic field.

\section{Future aspects}

This concept (morphogenetic field) will be tested, refined and added an exciting convergence of technologies. This model may work in:

- Finding easiest path towards gaining rational control over biological shapes of cells, tissues, organs and perhaps for entire organisms.

- Better understanding of histological behavior and the emergent properties of cells, tissues, organs and entire organisms with their concomitant morphology.

- Developing mathematical model for biological developmental patterning e.g. developmental pattern of malignant tumors.

- Choosing the strategies for advancing the field of regenerative medicine in cancer treatment.

- Helping to understand the underlying molecular mechanisms scraping the information for patterning of an organism.

- Discovering the inter connection between biological information and the geometrical form of cancer.

- Giving an object governing a law for decoding the biological information and transforming it into corresponding instructions for cell event depending on the positional information of a cancer cell.

- Observing the situations in which information is encoded from the CNS that is used for pattern control and cancer suppression.

\section{Conclusion}

The mechanisms behind cell organization and morphogenesis are very difficult to describe in terms of a conventional molecular biological frame. Without complete understanding about the cellular nature, it is not possible to understand the nature of cancer properly. Therefore, the new formulation of biology within the primacy of consciousness based on morphogenetic field concept is required. 


\section{References}

Aloisio R, Blasi P, Galante A, Ghia PL and Grillo AF (2004). Quantum-Gravity phenomenology and high energy particle propagation. arXiv preprint astro-ph/0410413.

Astigiano S, Damonte P, Fossati S, Boni L and Barbieri O (2005). Fate of embryonal carcinoma cells injected into postimplantation mouse embryos. Differentiation, 73: 484-90.

Beloussov LV, Louchinskaia NN and Stein AA (2000). Tension-dependent collective cell movements in the early gastrula ectoderm of Xenopus laevis embryos. Development Genes and Evolution, 210(2): 92-104.

Beloussov LV (2001). Morphogenetic Fields: Outlining the Alternatives and Enlarging. In Rivista di Biologia/Biology Forum, 94: 219-236.

Biskind MS and Biskind GR (1945). Tumor of rat testis produced by heterotransplantation of infantile testis to spleen of adult castrate. Proceedings of the Society for Experimental Biology and Medicine, 59(1): 4-8.

Biskind MS and Biskind GS (1944). Development of tumors in the rat ovary after transplantation into the spleen. Proceedings of the Society for Experimental Biology and Medicine, 55(3): 176-9.

Breedis C (1952). Induction of accessory limbs and of sarcoma in the newt (Triturus viridescens) with carcinogenic substances. Cancer Research, 12(12): 861-6.

Breivik J (2005). The evolutionary origin of genetic instability in cancer development. In: Seminars in Cancer Biology (Vol. 15, No. 1). Academic Press, pp. 51-60.

Brockes JP (1998). Regeneration and cancer. Biochim. Biophys. Acta, 1377: 1-1.

Costa M, Yan Y, Zhao D and Salnikow K (2003). Molecular mechanisms of nickel carcinogenesis: gene silencing by nickel delivery to the nucleus and gene activation/inactivation by nickel-induced cell signaling. Journal of Environmental Monitoring, 5(2): 222-3.

Discher DE, Janmey P and Wang YL (2005). Tissue cells feel and respond to the stiffness of their substrate. Science, 310(5751): 1139-43.

Furusawa C and Kaneko K (2003). Robust development as a consequence of generated positional information. Journal of Theoretical Biology, 224(4): 413-35.

Goldberg AD, Allis CD and Bernstein E (2007). Epigenetics: a landscape takes shape. Cell, 128(4): 635-8.

Goswami A and Todd D (1997). Is there conscious choice in directed mutation, phenocopies, and related phenomena? An answer based on quantum measurement theory. Integrative Physiological and Behavioral Sci., 32(2): 132-42.

Haldi M, Ton C, Seng WL and McGrath P (2006). Human melanoma cells transplanted into zebrafish proliferate, migrate, produce melanin, form masses and stimulate angiogenesis in zebrafish. Angiogenesis, 9(3): 139-51.

Hasan KM, Rahman MS, Arif KM and Sobhani ME (2012). Psychological stress and aging: role of glucocorticoids (GCs). Age 34(6): 1421-33.

Huang S and Ingber DE (2000). Shape-dependent control of cell growth, differentiation, and apoptosis: switching between attractors in cell regulatory networks. Experimental Cell Research, 261(1): 91-103.

Kampa M and Castanas E (2008). Human health effects of air pollution. Environmental pollution, 151(2): 362-7.

Kan Z, Zheng H, Liu X, Li S, Barber T, Gong Z, Gao H, Hao K, Willard MD and Xu J, Hauptschein R (2013). Whole genome sequencing identifies recurrent mutations in hepatocellular carcinoma. Genome Research, :154492.

Koestler A (1967). The ghost in the machine. Hutchinson (UK), MacMilan (USA), pp. 1-381.

Laszlo E (1996). The Systems View of the World a Holistic Vision for Our Time. Hampton Press. 
Leszczynski D, Joenväärä S, Reivinen J and Kuokka R (2002). Non-thermal activation of the hsp27/p38MAPK stress pathway by mobile phone radiation in human endothelial cells: molecular mechanism for cancer-and blood-brain barrier-related effects. Differentiation, 70(2-3): 120-9.

Levin M (2007). Large-scale biophysics: ion flows and regeneration. Trends in Cell Biology, 17(6): 261-70.

Levin M (2012). Morphogenetic fields in embryogenesis, regeneration, and cancer: non-local control of complex patterning. Biosystems, 109(3): 243-61.

Needham J (1936). New advances in the chemistry and biology of organized growth. Proc R Soc Med., 29(12): 1577-26.

Parsons DW, Li M, Zhang X, Jones S, Leary RJ, Lin JC, Boca SM, Carter H, Samayoa J, Bettegowda C and Gallia GL (2011). The genetic landscape of the childhood cancer medulloblastoma. Science, 331(6016): 435-9.

Popp FA (2003). Properties of biophotons and their theoretical implications. Indian J Exp Biol., 41(5): 391-402.

Prehn RT (1997). Regeneration versus neoplastic growth. Carcinogenesis, 18(8): 1439-44.

Reya T, Morrison SJ, Clarke MF and Weissman IL (2001). Stem Cells, Cancer and Cancer Stem Cells.Nature, 414(6859): 105.

Rhaman S, Islam F, Mamun A and Abdul-Awal SM (2014). Evolution of cancer: a quantum mechanical approach. Eur J Biophys., 2: 38-48.

Sahtouris E (1999). Earthdance: Living systems in evolution, pp. 1-432.

Schrödinger E (1958). Mind and matter. Cambridge University Press, pp. 1-104.

Schrödinger E (1944). What is life? the physical aspect of the living cell and mind. Cambridge University Press, Cambridge.

Sheldrake R (1992). An experimental test of the hypothesis of formative causation. Riv. Biol., 85: 431-43.

Sheldrake R (2005). New science of life. Icon Books Ltd.

Stevens LC (1970). The development of transplantable teratocarcinomas from intratesticular grafts of pre-and postimplantation mouse embryos. Developmental Biology, 21(3): 364-82.

Sun H, Shamy M, Costa M (2013). Nickel and epigenetic gene silencing. Genes, 4(4): 583-95.

Whitehead AN (2012). Nature and life. Cambridge University Press. 